\title{
Pathophysiological and Pharmacological Rationale for the Use of Exenatide Once Weekly in Patients with Type 2 Diabetes
}

Samuel S. Grossman

To view enhanced content go to www.advancesintherapy.com Received: January 10, 2014 / Published online: February 18, 2014

(c) The Author(s) 2014. This article is published with open access at Springerlink.com

\section{ABSTRACT}

Introduction: A new formulation of exenatide has become available recently that is the first antidiabetic medication for type 2 diabetes mellitus (T2DM) dosed on a weekly schedule. This review summarizes the pharmacology, efficacy, and safety of exenatide once weekly (exenatide QW). The results are interpreted in terms of the pathophysiology of T2DM, as well as the pharmacology of the new formulation.

Methods: Relevant literature on exenatide QW and diabetes was identified through PubMed database searches from inception until September 2013.

Discussion: In the new once-weekly formulation of exenatide, the exenatide molecule is dispersed in microspheres. Following subcutaneous injection, these microspheres degrade in situ and slowly release active agent. In clinical trials, therapy

Electronic supplementary material The online version of this article (doi:10.1007/s12325-014-0101-4) contains supplementary material, which is available to authorized users.

S. S. Grossman $(\bowtie)$

Diabetes Care On-The-Go Inc., Edison, NJ, USA

e-mail: samfordiabetes@gmail.com with exenatide QW as monotherapy or in combination with other antidiabetic treatments was associated with reductions in glycated hemoglobin $(-1.3 \%$ to $-1.9 \%)$, fasting plasma glucose ( -32 to $-41 \mathrm{mg} / \mathrm{dL}$ ), and body weight $(-2.0$ to $-3.7 \mathrm{~kg}$ ). These outcomes were achieved without an associated increase in the rate of hypoglycemic episodes, except when exenatide QW was used in combination with sulfonylureas. The primary tolerability issues in the trials were gastrointestinal adverse events, particularly during the first weeks of use, although the rate of nausea during startup with exenatide QW was lower than that with the related agents, exenatide twice daily and liraglutide once daily.

Conclusions: Exenatide QW may be particularly well suited to patients who desire the benefits associated with glucagon-like peptide-1 receptor agonists, including significant glycemic control, low risk of hypoglycemia, and moderate weight loss, but prefer the convenience of once-weekly dosing.

Keywords: Endocrinology; Exenatide once weekly; GLP-1 receptor agonists; Incretin therapy; Type 2 diabetes mellitus 


\section{INTRODUCTION}

Multiple pathological derangements contribute to the chronic hyperglycemia and hypertriglyceridemia that characterize type 2 diabetes mellitus (T2DM) [1-3]. Although specific details may vary in individual patients, these pathological defects can occur in the following organs and tissues: skeletal muscle, which becomes less effective at absorbing glucose after a meal; liver, which releases too much glucose into the blood during fasting; pancreatic beta-cells, which fail to produce enough insulin to maintain normoglycemia (despite overexpressing the hormone relative to healthy patients); pancreatic alpha-cells, which secrete excess glucagon; adipose tissue, which secretes excess lipids; the gastrointestinal tract, which secretes reduced levels of incretin factors; and the kidney, which reabsorbs too much glucose (Fig. 1). All told, these derangements combine to drive up blood sugar and serum lipids and, in so doing, dramatically raise the risk of macrovascular and microvascular events.

Despite the complexities of this clinical picture, the pathophysiological defects underlying T2DM can be attributed in large part to a single core defect, that is, inappropriate resistance to the biologic effects of insulin [1, 2]. The mechanism by which insulin resistance develops in various tissues, a process that involves alterations in cellular signal transduction pathways and receptors/ transporters in the affected tissues [4], is beyond the scope of this review. Nonetheless, it is important to note that one of the key features of insulin resistance is its progression over time [5], which results in beta-cells producing increasingly more insulin to
Incretin therapy, insulin, SFUs, TZDs

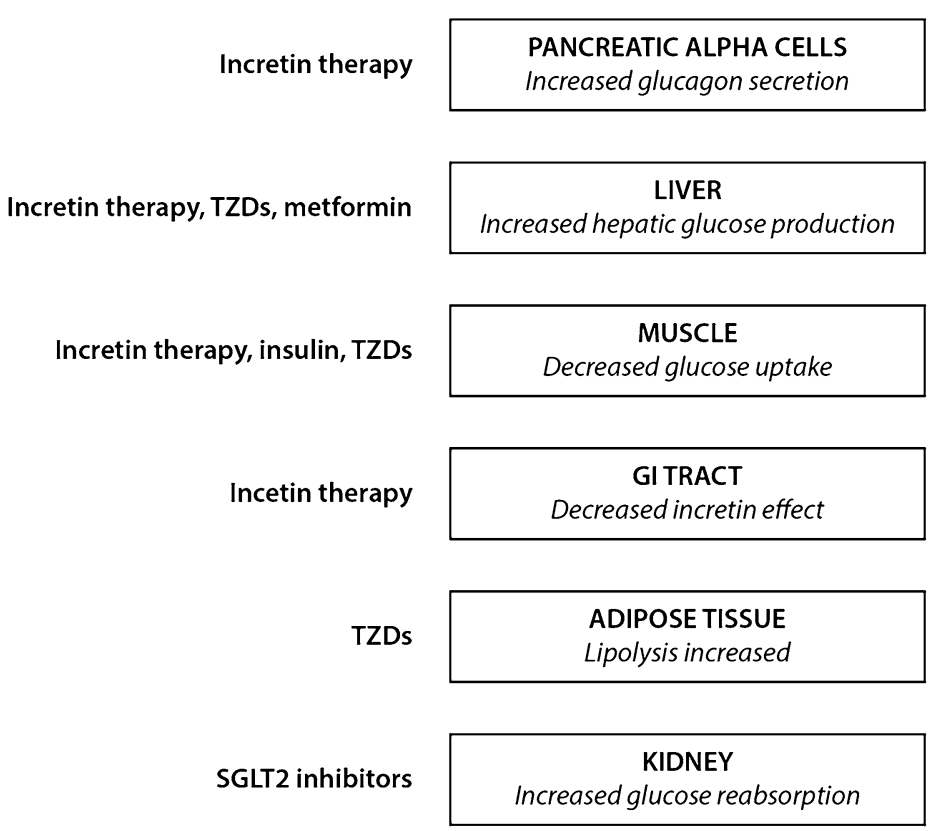

Insufficient insulin secretion
PANCREATIC BETA CELLS

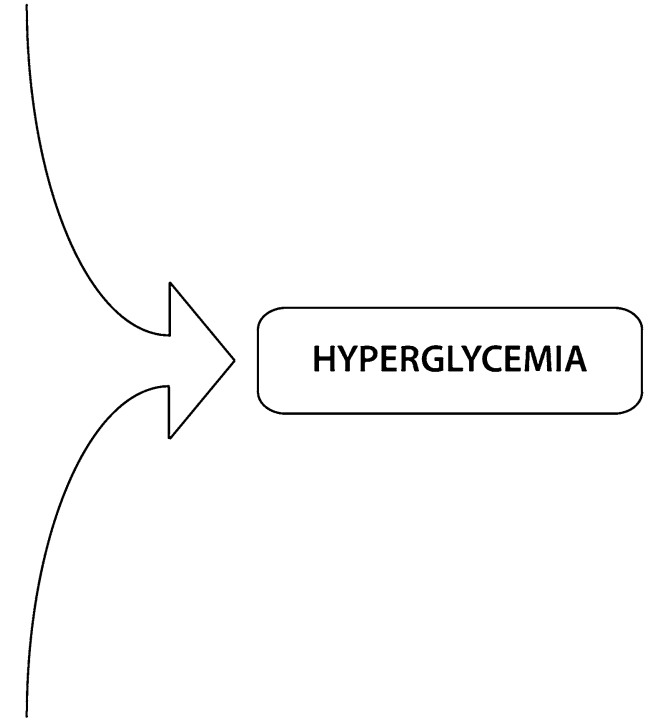

Fig. 1 Pathophysiologic disturbances in T2DM that may lead to hyperglycemia and the sites of action of antidiabetes medications. Incretin therapy includes glucagon-like peptide- 1 receptor agonists and dipeptidyl peptidase- 4 inhibitors 
compensate. Ultimately, however, because their synthetic capacity becomes exhausted, because they are damaged by increased levels of serum lipids, or for reasons as yet unknown [6], betacells eventually lose the ability to produce sufficient insulin, and insulin replacement therapy may become necessary.

Managing this complex and evolving clinical picture can be extremely challenging. The most commonly used antidiabetic medications include metformin, sulfonylureas, thiazolidinediones, incretin therapies [glucagon-like peptide-1 receptor agonists (GLP-1RAs) and dipeptidyl peptidase-4 (DPP-4) inhibitors], sodium-glucose co-transporter 2 (SGLT2) inhibitors, and basal and bolus insulins [7]. Three treatment approaches have been published recently that recommend how to combine these therapies to achieve optimal glucose control $[3,7,8]$. All recommend that therapeutic regimens for T2DM should be chosen, in part, based on an understanding of the underlying pathophysiology. In particular, they recommend medications for polytherapy that complement one another, i.e., have different targets among the organs and tissues most affected in T2DM. Fortunately, the armamentarium of available antidiabetes medications now contains individual agents that target virtually all of these organs and tissues (Fig. 1).

\section{METHODS}

This review will examine the role of one class of antidiabetes medications, the GLP-1RAs, with a particular focus on the newest member of the class, exenatide once weekly (exenatide QW). It will attempt to rationalize exenatide QW use in terms of the underlying pathology and its pharmacology. The potential value of the new once-weekly treatment modality will also be discussed. Relevant literature was identified through PubMed database searches from inception until September 2013. Search terms included exenatide, glucagon-like peptide-1, GLP-1 receptor agonists, type 2 diabetes mellitus, diabetes pathophysiology, and other terms specifically associated with individual sections of the review.

\section{Compliance with Ethics}

The analysis in this article is based on previously conducted studies and does not involve any new studies of human or animal subjects performed by the author.

\section{DISCUSSION}

\section{Pharmacology}

Three GLP-1RAs, exenatide twice daily (exenatide BID) [9], liraglutide once daily (liraglutide QD) [10], and exenatide QW [11] are currently available in the USA for treating T2DM. All three lower blood glucose by replicating the activity of GLP-1, a naturally occurring hormone released from intestinal L cells in response to a meal [12, 13]. Native GLP-1 acts in an endocrine and/or paracrine fashion to restore euglycemia by multiple mechanisms. These mechanisms include stimulating insulin secretion from pancreatic beta-cells [12]; inhibiting glucagon secretion from pancreatic alpha-cells in the presence of elevated blood glucose levels [14, 15]; decreasing gastric motility to slow nutrient absorption [16]; and inducing feelings of satiety [17] (Fig. 1).

Exenatide is a 39-amino acid synthetic version of exendin-4, a peptide isolated 
from the lizard Heloderma suspectum [18]. The drug shares $53 \%$ sequence identity with human GLP-1 [13] and consequently binds with high affinity to GLP-1 receptors, thereby inducing all of its known glucoregulatory activities [19-21]. However, exenatide is more resistant to the activity of DPP-4 [18], a widely dispersed protease that rapidly cleaves native GLP-1 in vivo [22]. The first exenatide formulation manufactured for clinical use, known now as exenatide BID, remains at therapeutic concentrations within the bloodstream long enough to allow for twice-daily subcutaneous administration before the two main meals of the day [9]. Liraglutide QD, a modified form of mammalian GLP-1 that contains an amino acid substitution at position 34 and an added C16 palmitoyl fatty-acid side chain at position 26, remains at therapeutic concentrations long enough in vivo-with an elimination half-life of approximately $13 \mathrm{~h}$, in part because it is injected at very high concentrations (1.2 or $1.8 \mathrm{mg}$ per administration)_to allow for once-daily administration [10].

In the new once-weekly formulation of exenatide, the exenatide molecule is dispersed in microspheres $[11,23-26]$. Following subcutaneous administration, these microspheres undergo spontaneous hydrolysis into lactic and glycolic acids, which are easily eliminated as carbon dioxide and water. During this process, active drug is slowly released into circulation [23]. The combined release of exenatide from multiple once-weekly injections results in consistent blood concentrations over time, well above the level demonstrated to impart full pharmacologic effect [27].

\section{Efficacy}

Six randomized controlled clinical trials, known by the acronym DURATION (Diabetes Therapy Utilization: Researching Changes in A1c, Weight and Other Factors Through Intervention with Exenatide Once Weekly), have been conducted on exenatide QW, each lasting 24-30 weeks [28-33] (Table 1). In total, 3,225 patients with T2DM were assessed, of whom 1,379 received exenatide QW and 1,846 received comparators. Comparators included metformin, pioglitazone, sitagliptin, insulin glargine, exenatide BID, and liraglutide QD. In the trials, exenatide QW was assessed either as monotherapy or in combination with metformin, sulfonylureas, thiazolidinediones, or combinations of these agents.

\section{Exenatide QW Monotherapy}

Based on its relative cost compared with antidiabetic agents available generically, as well as its subcutaneous route of administration, exenatide QW may not be commonly thought of as first-line monotherapy, although indicated as an adjunct to diet and exercise in adults with T2DM [11]. This is consistent with the recent Standards of Medical Care in Diabetes from the American Diabetes Association, which recommend first-line metformin followed by optional second-line treatment with a GLP-1RA [7]. A second algorithm for antidiabetes therapy, known as the "pathophysiologic approach," recommends avoiding monotherapy altogether, instead advocating initiation with a combination of metformin, a thiazolidinedione, and a GLP-1RA [3]. The American Association of Clinical 


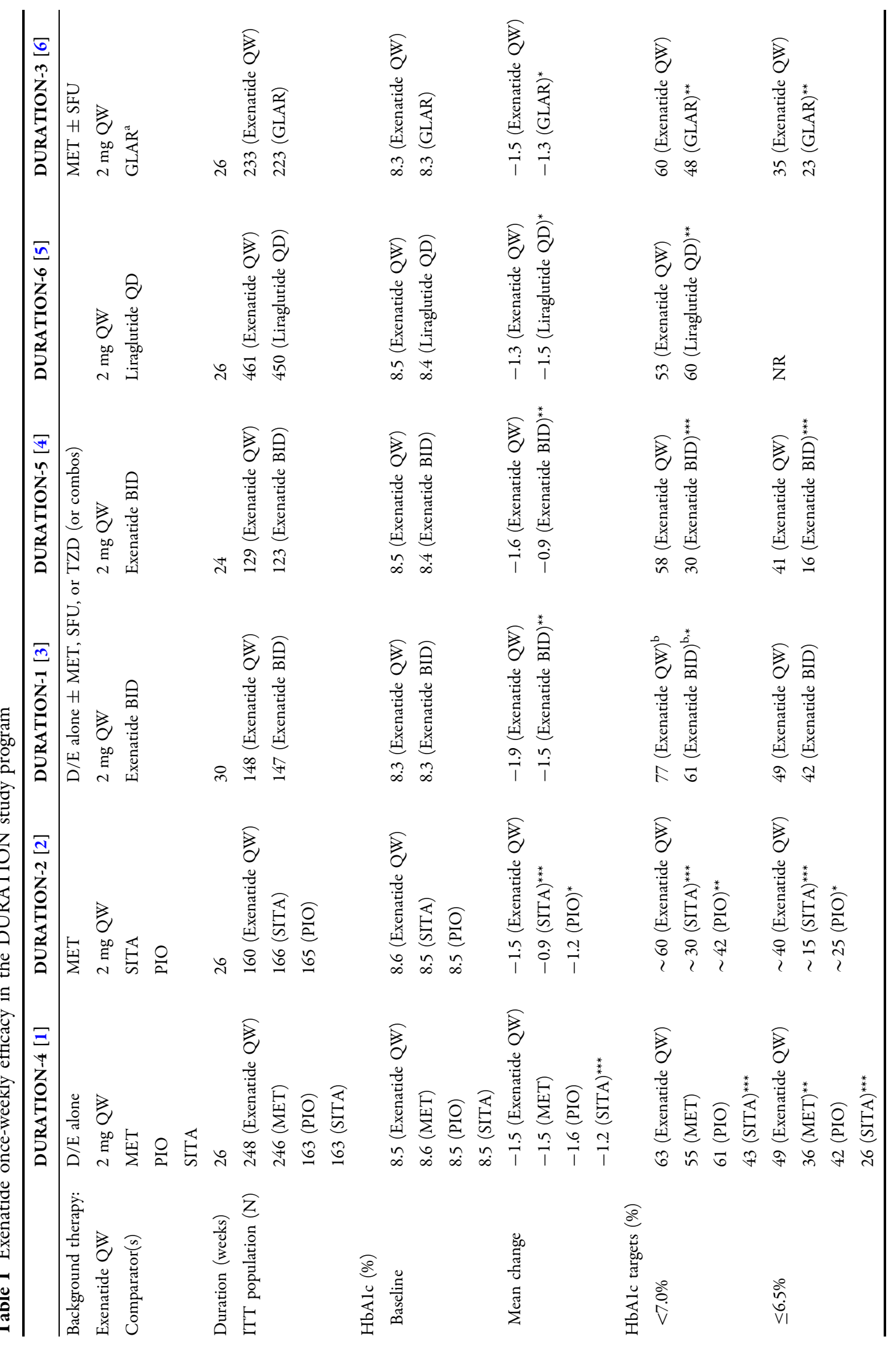




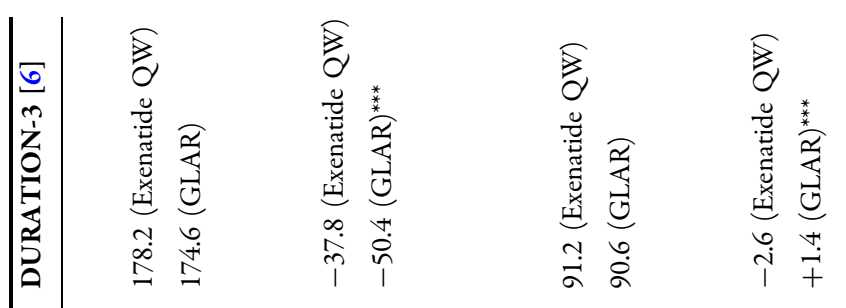

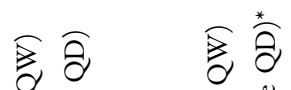

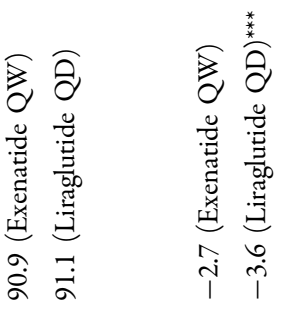

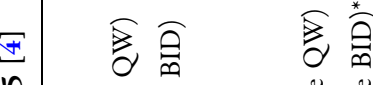

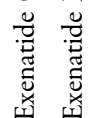
离离
i ?

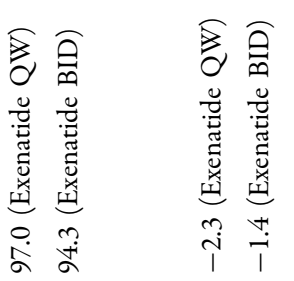
ก $\cong$
竞
高
苛
造这
莲華

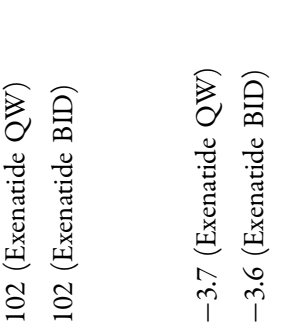

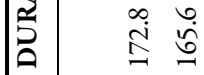

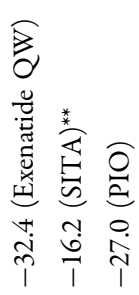

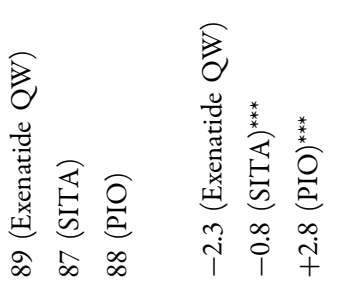

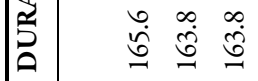

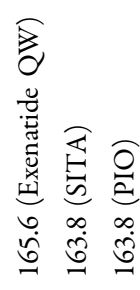
章
学

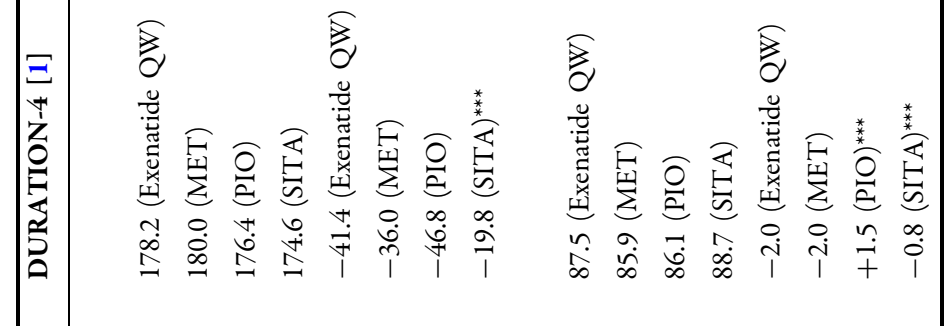

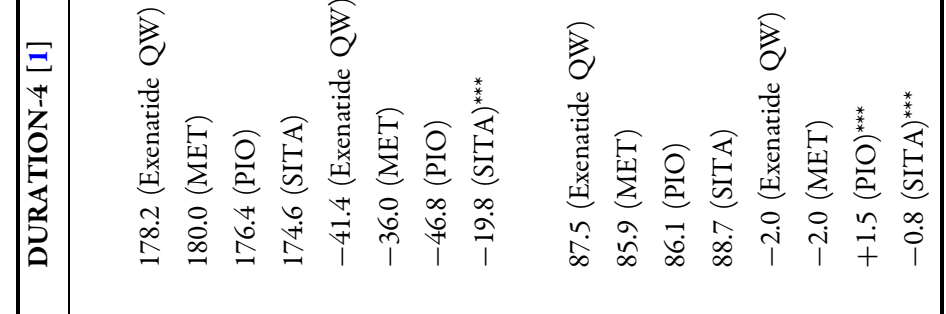

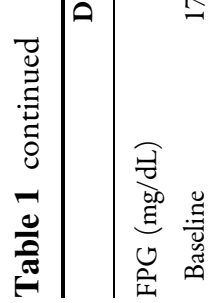

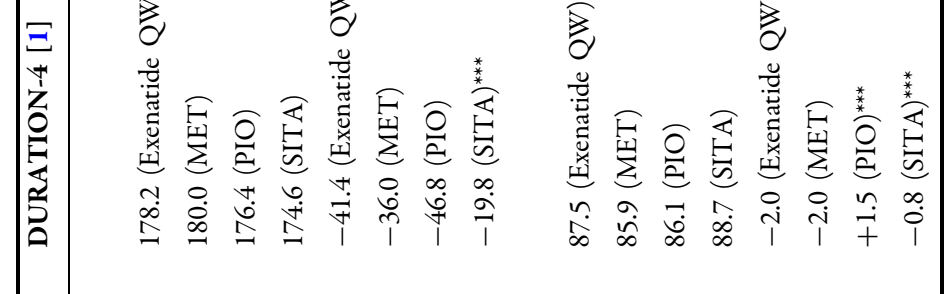

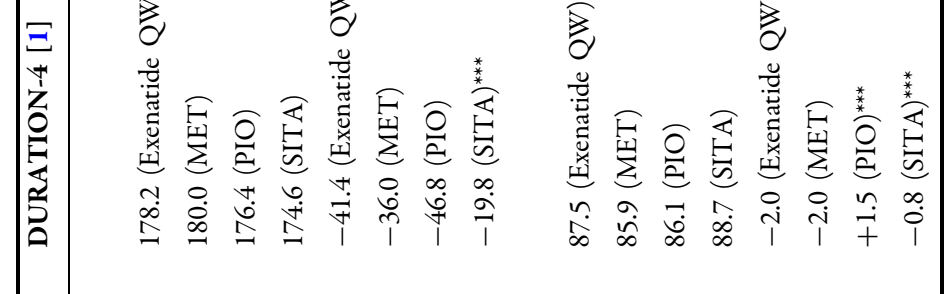

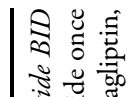
है:

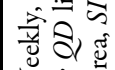
药

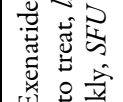
营苛

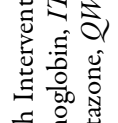

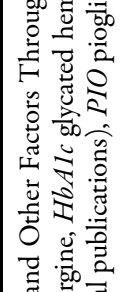

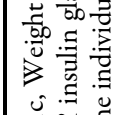

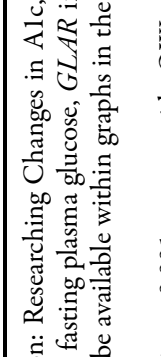

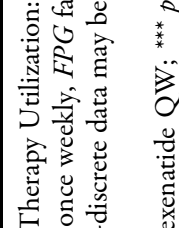

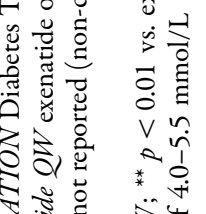

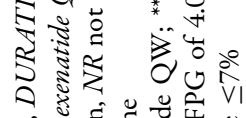

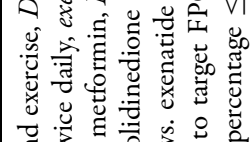

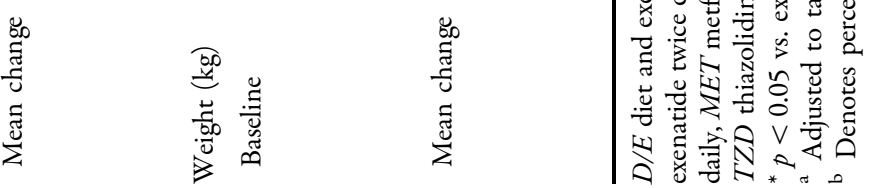


Table 2 Rates of gastrointestinal adverse events associated with incretin therapies in the DURATION study program

\begin{tabular}{lllll}
\hline & Nausea (\%) & Diarrhea (\%) & Vomiting (\%) & Constipation (\%) \\
\hline DURATION-1 & & & & 10.8 \\
Exenatide QW & 26.4 & 13.5 & 10.8 & 6.2 \\
Exenatide BID & 34.5 & 13.1 & 18.6 & 6 \\
DURATION-2 & & & & 2 \\
Exenatide QW & 24 & 18 & 11 & \\
Sitagliptin & 10 & 10 & 2 & 2.5 \\
DURATION-4 & & & & 2.5 \\
Exenatide QW & 11.3 & 10.9 & $\mathrm{NR}$ & $\mathrm{NR}$ \\
Sitagliptin & 3.7 & 5.5 & $\mathrm{NR}$ & $\mathrm{NR}$ \\
DURATION-5 & & & 4.7 & 4.6 \\
Exenatide QW & 14.0 & 9.3 & 8.9 & 4.9 \\
Exenatide BID & 35.0 & 4.1 & & \\
DURATION-6 & & 6.1 & 10.7 & \\
Exenatide QW & 9.3 & 13.1 & 3.7 & \\
Liraglutide QD & 20.7 & &
\end{tabular}

DURATION Diabetes Therapy Utilization: Researching Changes in A1c, Weight and Other Factors Through Intervention with Exenatide Once Weekly, exenatide BID exenatide twice daily, exenatide $Q W$ exenatide once weekly, $N R$ not reported

Endocrinologists (AACE) indicates that GLP1RAs are appropriate options as monotherapy in patients who start pharmacotherapy with an entry glycated hemoglobin (Hb)A1c <7.5\%; in patients who start initial pharmacotherapy at higher HbA1c levels, the GLP-1RAs are recommended as first options in dual therapies (entry HbA1c $\geq 7.5 \%$ ) or triple therapies (entry HbA1c $\geq 9.0 \%)$ [8].

The DURATION-4 study directly compared exenatide QW monotherapy with metformin monotherapy and pioglitazone monotherapy (an additional arm with sitagliptin will be described in the next section). Results showed that long-term control appeared similar for all three agents [32] (Table 1). Thus, in patients suboptimally controlled on diet and exercise, changes from baseline in HbA1c after 26 weeks of therapy with exenatide QW, metformin, or pioglitazone were $-1.5 \%,-1.5 \%$, and $-1.6 \%$, respectively ( $p=\mathrm{NS}$ for all comparisons). Similarly, changes from baseline in fasting plasma glucose (FPG) were, respectively, $-41.4, \quad-36.0$, and $-46.8 \mathrm{mg} / \mathrm{dL} \quad(p=$ not significant (NS) for all comparisons). Therapy with exenatide QW, however, appeared to be distinguishable by other effects. For example, exenatide QW, unlike metformin, significantly slowed down the time-dependent loss of glucose control in T2DM. This was shown in an extension study of DURATION-3, which demonstrated that patients on exenatide QW maintained most of their $\mathrm{HbA1c}$ reductions for at least 3 years [34]. Moreover, therapy with exenatide QW resulted in moderate weight loss (Table 1). 


\section{Exenatide QW Combination Therapy}

As described above, current guidelines recommend that polytherapy for T2DM should consist of individual agents that act in concert on different aspects of the underlying pathophysiology. Implicit in this recommendation is the notion that hyperglycemia in T2DM arises from accumulated incremental effects of dysregulation across all of the involved organs and tissues. Consequently, addressing as many pathophysiological problems as possible would be assumed, according to this model, to have the greatest impact on blood sugar. The most direct test of this assumption would be to compare outcomes in treatment-naive subpopulations started on one, two, three, or more therapies. Only a few studies with GLP1RAs have taken this, or a similar, approach [35-37].

Most drug trials have taken a different approach. Their basic design involves administering the test agent of interest to a population of patients who are no longer achieving optimal glucose control on a preexisting regimen of background therapy. Again, this would be expected to successfully lower blood glucose only if the individual agents that compose the regimen act, at least partially, on independent targets. DURATION-1, $-2,-3,-5$, and -6 have all taken this approach [28-31, 33], and in each case, the addition of exenatide QW to suboptimal background therapy significantly improved glycemic parameters (Table 1). Thus, in general, the addition of exenatide QW to other classes of antidiabetic medications provided significant add-on therapeutic benefit in T2DM.

Several other conclusions can be drawn from the results of the DURATION study program. First, although similar in many respects, exenatide QW and exenatide BID should not be considered directly interchangeable. DURATION-1 [31] and DURATION-5 [29] examined the effects of these two agents on patients who were achieving suboptimal control on diet and exercise alone \pm metformin, a sulfonylurea, a thiazolidinedione, or a combination of the prior agents. In DURATION-1, exenatide QW was associated with greater reductions than exenatide BID in HbA1c ( $-1.9 \%$ vs. $-1.5 \%$, $p=0.0023)$ and FPG (-41.4 vs. $-25.2 \mathrm{mg} / \mathrm{dL}$, $p<0.0001)$. Conversely, reductions from baseline in 2-h postprandial glucose (PPG) levels were significantly greater with exenatide BID than exenatide QW. Similar observations were made in DURATION-5. These results have led some to suggest that the twice-daily formulation, which has its strongest effect on PPG, is a particularly ideal "fit" with basal insulin, which primarily affects FPG [38]. The hypothesis was confirmed in a recently published randomized clinical trial, which showed that the addition of exenatide BID to titrated basal insulin provided greater glycemic control than titrated basal insulin alone, and did so without an increase in hypoglycemic events and with modest weight loss [39]. Studies are currently ongoing to examine the effect of exenatide QW in combination with basal insulin.

Another study directly compared exenatide QW and titrated basal insulin, and the results constitute a second key finding of the DURATION study program. In DURATION-3 [30], patients inadequately controlled on background metformin \pm a sulfonylurea exhibited greater reductions in HbA1c $(-1.5 \%$ vs. $-1.3 \% ; p=0.0003)$, but smaller reductions in FPG ( -37.8 vs. $-50.4 \mathrm{mg} / \mathrm{dL} ; p=0.001)$, after 26 weeks of therapy with exenatide $\mathrm{QW}$ relative to basal insulin. After 3 years of therapy, HbA1c 
reductions remained greater in the exenatide QW group ( $-1.01 \%$ vs. $0.81 \% ; p=0.03)$ and FPG reductions remained less $(-31.1$ vs. $47.7 \mathrm{mg} / \mathrm{dL} ; \quad p<0.001) \quad$ [34]. Patients on exenatide QW also exhibited weight loss, whereas those on basal insulin exhibited weight gain (Table 1). Thus, from an efficacy perspective, long-term use of exenatide QW was at least as effective as basal insulin. This result may appear unexpected at first, given that therapeutic insulin can be dosed to produce virtually any desired blood glucose value $[1,2]$. However, intensive insulin therapy is limited by concerns over hypoglycemic episodes, which correlate with overall mortality [40-42]; hypoglycemia is not as great a concern with exenatide QW (see "Safety", below).

A third key point from the DURATION study program is that exenatide QW and the DPP-4 inhibitor sitagliptin did not have identical activities, despite the fact that both agents come under the general rubric of incretin therapies. Both as monotherapy and in combination with metformin, exenatide QW was associated with greater reductions in HbA1c, FPG, and weight than sitagliptin (Table 1). For instance, in the 26-week DURATION-4 monotherapy trial [32], the exenatide QW and sitagliptin groups experienced reductions in HbA1c of $-1.5 \%$ and $-1.2 \%$, respectively $(p<0.001)$; in FPG of -41.4 and $-19.8 \mathrm{mg} / \mathrm{dL}(p<0.001)$; and in weight of $-2.0 \mathrm{~kg}$ and $-0.8 \mathrm{~kg}(p<0.001)$. This likely reflects the underlying pharmacology of the two agents. Pharmacokinetic experiments have demonstrated that overall exposure to endogenous GLP-1 increases approximately twofold in patients on DPP-4 inhibitors and that blood hormone levels fluctuate up and down between postprandial and fasting states, similar to what occurs in drug-naive healthy individuals [43]. By contrast, repeated administrations of exenatide QW resulted in essentially constant and high levels of active agent [27]. Thus, in thinking how to distinguish between the two agents, clinicians should consider the clinical effect on HbA1c and weight, as well as tolerability profile and administration route.

Finally, in DURATION-6 [33], exenatide QW appeared to have lower efficacy than liraglutide QD. Across the 26-week study period in the exenatide QW and liraglutide QD groups, HbA1c decreased by $-1.3 \%$ and $-1.5 \%$, respectively $(p=0.02)$; FPG decreased by -31.7 and $-38.2 \mathrm{mg} / \mathrm{dL} \quad(p=0.02)$; and weight decreased by -2.7 and $-3.6 \mathrm{~kg}$ $(p=0.005)$. As with the exenatide QW and sitagliptin comparison, a clinician choosing between exenatide QW and liraglutide QD should consider clinical efficacy, but also dosing convenience and tolerability (see Safety, below).

\section{Safety}

The bulk of safety data on exenatide QW derives from the DURATION study program, which consisted of trials lasting between 24 and 30 weeks. The information provided in this section summarizes these data, but it should be borne in mind that longer-term side effects, if any, remain to be determined.

\section{Gastrointestinal Effects}

Gastrointestinal side effects are the primary tolerability issue with GLP-1RAs, including exenatide QW (Table 2). In the DURATION study program, the most common gastrointestinal adverse event, mild to moderate nausea, occurred at rates ranging from $9.3 \%$ to $26.4 \%$ across all studies. Reported cases of nausea occurred 
predominantly in the first 6-8 weeks of therapy and tapered off significantly thereafter [23]. In direct head-to-head comparisons, exenatide QW was associated with significantly less nausea than either exenatide BID or liraglutide QD. In DURATION-6, rates of nausea in patients on exenatide QW and liraglutide QD were 9.3\% and 20.7\%, respectively. The greater gastrointestinal tolerability of exenatide QW relative to the other GLP-1RAs likely reflects its pharmacokinetic properties, specifically the more gradual rise in active agent in the blood that occurs after initiating the once-weekly formulation relative to other GLP-1RAs [44].

\section{Hypoglycemia}

No episodes of major hypoglycemia were documented in patients on exenatide QW in any of the DURATION trials [28-33]. Moreover, the incidence of minor hypoglycemia (defined as a plasma glucose concentration $<54 \mathrm{mg} / \mathrm{dL}$ ) was $2.0 \%$ in patients who received exenatide QW as monotherapy or in combination with metformin or a thiazolidinedione (Amylin Pharmaceuticals, data on file). These results are consistent with the known properties of exenatide vis-à-vis insulin secretion. In hyperinsulinemic-hypoglycemic clamp experiments, intravenous exenatide was shown to maximally stimulate insulin secretion when blood glucose was above $90 \mathrm{mg} / \mathrm{dL}$ ( $5 \mathrm{mmol} / \mathrm{L}$ ) [45]. As blood glucose declined below this level toward the euglycemic range, insulin secretory rates declined as well, eventually becoming indistinguishable from those of the control placebo patients when glycaemia reached $72 \mathrm{mg} / \mathrm{dL} \quad(4 \mathrm{mmol} / \mathrm{L})$. In other words, exenatide-induced insulin secretion is glucose dependent and, thus, occurs mainly in the presence of hyperglycemia. By contrast, insulin and insulin secretagogues, such as sulfonylureas, continue to reduce blood sugar even after blood glucose has fallen into the hypoglycemic range. Consequently, in clinical trials, sulfonylureas in combination with exenatide QW were observed to result in higher levels of hypoglycemia [23]. In patients receiving this combination, it is therefore recommended that the sulfonylurea dosage be carefully monitored and reduced if necessary [11].

\section{Cardiovascular Effects}

Ever since a potential association was observed between rosiglitazone use and elevated cardiovascular (CV) risk [46], as well as between intensive antidiabetic therapy and adverse cardiac outcomes in some patients [47], all antidiabetic medications have come under increased surveillance for CV safety, including long-term post-marketing safety studies [48]. Exenatide QW is currently undergoing such studies, although the results are not yet available. Definitive conclusions must await the results, but three current observations suggest a significant $\mathrm{CV}$ signal may not be expected. First, clinical studies on exenatide at therapeutic and supra-therapeutic concentrations did not prolong QTc interval in healthy subjects [49]. A similar lack of effect was observed in another trial on exenatide QW [50]. Second, in multiple DURATION studies, exenatide QW therapy was associated with moderate improvements in systolic blood pressure and blood lipids [28-33]. Third, metaanalyses of short-term studies with exenatide BID identified no increased CV risk [51].

\section{Pancreatic Effects}

The potential association between incretin therapies, both GLP-1RAs and DPP-4 
inhibitors, and pancreatic effects has received significant attention recently $[52,53]$. The concern initially came to light in a published work that found elevated rates of spontaneously reported pancreatitis and pancreatic cancer in the US Food and Drug Administration's (FDA's) Adverse Event Reporting System among patients on incretin therapies [54]. This analysis, however, has come under significant criticism for its dependence on uncontrolled and spontaneously reported events and for its bias in focusing exclusively on pancreatic events. A subsequent analysis of the same database found that whereas spontaneous reports of pancreatic cancer were indeed higher, reports of several other cancers were significantly lower in incretin users [55]. Interpretations of the data are further complicated by the fact that T2DM per se is associated with increased risk of pancreatitis. A retrospective study of diabetic patients $(n=337,067)$ and age- and sex-matched nondiabetic patients $(n=337,067)$ found a pancreatitis incidence rate of 4.22 cases per 1,000 patient-years in the diabetic cohort versus 1.49 cases per 1,000 patient-years in the nondiabetic cohort [56]. Pancreatitis risk may also be increased in the setting of obesity, another common complication of T2DM [57].

Ultimately, the potential role, if any, of incretin therapies in pancreatic side effects will require long-term post-marketing observational trials designed to directly assess this question. These studies are ongoing for multiple incretin therapies, and results have begun to be reported. The results appear encouraging. The rates of pancreatitis among patients on the DPP4 inhibitors saxagliptin and alogliptin were statistically indistinguishable from those among patients on other antidiabetic agents $[58,59]$. Similar prospective data have not yet been reported for GLP-1RAs, but a retrospective study of a large insurance claims database came to a similar conclusion for exenatide [60]. The absolute risk of acute pancreatitis among exenatide initiators was $0.13 \%$ (37 cases among 27,995 patients followed for up to 1 year), which was equivalent to the absolute risk in a propensity score-matched cohort of metformin/glyburide initiators $(0.13 \%$; 36 cases per 27,983 patients).

A report from the European Medicines Agency concluded that present data do not confirm recent concerns over an increased risk of pancreatic adverse events with GLP-1-based therapies [61]. The FDA concurred in this conclusion [62]. Exenatide QW has not been studied in patients with a history of pancreatitis, however [11]. Consequently, until prospective studies become available, it is prudent to follow the recommendations on the package insert, i.e., discontinue therapy with exenatide QW in patients with pancreatitis and consider other classes of antidiabetic medications in patients with a history of pancreatitis.

\section{Use in Patients with Renal Impairment and the Elderly}

Managing systemic therapy in the setting of renal impairment, which is associated with both increasing duration of T2DM and age, is a significant challenge. Nonclinical studies have shown that exenatide is predominantly eliminated by glomerular filtration [11, 63], making this issue one of particular relevance to patients on exenatide QW. In clinical trials, severe nausea, severe vomiting, and rapidly declining blood glucose concentrations, including severe hypoglycemia requiring parenteral glucose administration, were associated with overdoses of exenatide BID [11], although it 
should be noted that overdose is extremely unlikely with the single-use administration device for exenatide QW.

Based on the prior considerations, exenatide QW should not be used in patients with severe renal impairment (creatinine clearance $<30 \mathrm{~mL}$ / min) or end-stage renal disease. As the injector apparatus for exenatide QW comes prepackaged with a single, fixed amount of agent for subcutaneous administration, decreasing its dosage is not an option. Instead, in patients on exenatide QW who have mild to moderate renal impairment (creatinine clearance 30-50 mL/ min), down-titration of concomitant medications, in particular sulfonylureas, may become necessary to manage hypoglycemia and other adverse events.

Post-marketing case reports of worsened kidney function associated with exenatide use have raised concern over a causal relationship [11]. However, as with the potential pancreatic effects described above, drawing conclusions from spontaneous reports is highly problematical. A recent meta-analysis examined six randomized trials (16-30 weeks) comparing exenatide BID $(n=905)$ with placebo $(n=916)$ and two similar trials (24-30 weeks) comparing exenatide BID $(n=268)$ with exenatide QW $(n=277)$ [64]. Overall, across the trials, the decline in glomerular filtration rates did not differ between the comparators. Thus, to date, a claim of a causal link between exenatide use and worsening kidney function remains unsupported. This is consistent with another analysis, which failed to find any clinically significant differences among elderly patients who participated in the DURATION study program [65], although it is important to bear in mind that the trials excluded patients with severe renal impairment.

\section{Adherence and Cost}

Exenatide QW was formulated to increase patient convenience by decreasing the frequency of injections. However, no postmarketing studies have yet assessed whether this added convenience translates into higher treatment compliance. In general terms, though, studies have confirmed that adherence to antidiabetic regimens remains unsatisfactory [66] and that ease of administration results in higher treatment compliance [67]. A more directly relevant study surveyed 1,516 adults with T2DM and assessed their perceptions of a once-weekly medication [68]. The results showed that positive attitudes regarding a once-weekly dosing regimen were common, with beneficial aspects perceived to include greater convenience, better medication adherence, improved quality of life, and a less overwhelming sense of treatment $(p<0.001$ for all comparisons). Approximately, $47 \%$ of surveyed patients reported they would likely take an injectable once-weekly medication if recommended by their physician, with current injection users more than twice as likely as non-injection users $(73.1 \%$ vs. $31.5 \%$; $p<0.001)$.

Cost-benefit analyses have been performed for exenatide QW relative to exenatide BID [69], insulin glargine [69-72], sitagliptin [73], and pioglitazone [73]. In general, exenatide QW was predicted to be cost effective relative to insulin glargine, as measured by quality-adjusted life years, in the healthcare systems of the UK, Spain, Switzerland, and the USA [69-72]. Similarly, in a validated computer model, exenatide QW was projected to improve health and reduce lifetime costs for diabetesrelated complications compared with sitagliptin or pioglitazone [73]. 


\section{CONCLUSION}

It is currently recommended that optimal antidiabetic polytherapy should use therapeutic agents that act in concert on different aspects of T2DM. By this criterion, exenatide QW, like other GLP-1RAs, is an attractive option, as it affects multiple organs and tissues involved in the etiology of the disease. An increasing body of literature has demonstrated that exenatide QW provides reductions in $\mathrm{HbA1c}$ with little risk of hypoglycemia, and does so while simultaneously providing moderate weight loss. The primary safety issues associated with its use are gastrointestinal side effects, whereas other risks of recent concern, including $\mathrm{CV}$, pancreatic, and kidney effects, appear small and manageable based on available evidence. In general, the efficacy of exenatide QW appeared to be greater than that of the DPP- 4 inhibitor sitagliptin [28, 32] and slightly less than that of the GLP-1RA liraglutide QD [37]. On the other hand, the incidence of gastrointestinal events of exenatide QW appeared to be lower than that of liraglutide QD and higher than that of sitagliptin. Thus, exenatide QW may be particularly well suited to patients who desire the benefits associated with glucagon-like peptide-1 receptor agonists, including significant glycemic control, low risk of hypoglycemia, and moderate weight loss, but prefer the convenience of once-weekly dosing.

\section{ACKNOWLEDGMENTS}

The author would like to acknowledge David Norris PhD (Ecosse Medical Communications, Falmouth, MA, USA) for editorial assistance, which was supported by the AstraZeneca/ Bristol-Myers Squibb Diabetes Alliance.
Sponsorship and article processing charges for this study were funded by AstraZeneca.

The author meets the ICMJE criteria for authorship for this manuscript, takes responsibility for the integrity and the accuracy of the data analysis, and has given final approval for the version to be published.

Conflict of interest. SS Grossman serves on the Speakers Bureau of Becton, Dickinson and Company. He has no other conflicts of interest to disclose.

Compliance with ethics guidelines. The analysis in this article is based on previously conducted studies and does not involve any new studies of human or animal subjects performed by any of the authors.

Open Access. This article is distributed under the terms of the Creative Commons Attribution Noncommercial License which permits any noncommercial use, distribution, and reproduction in any medium, provided the original author(s) and the source are credited.

\section{REFERENCES}

1. DeFronzo RA. Lilly lecture 1987. The triumvirate: beta-cell, muscle, liver. A collusion responsible for NIDDM. Diabetes. 1988;37:667-87.

2. DeFronzo RA. Banting Lecture. From the triumvirate to the ominous octet: a new paradigm for the treatment of type 2 diabetes mellitus. Diabetes. 2009;58:773-95.

3. DeFronzo RA, Eldor R, Abdul-Ghani $M$. Pathophysiologic approach to therapy in patients with newly diagnosed type 2 diabetes. Diabetes Care. 2013;36(Suppl 2):S127-38.

4. Kashyap SR, DeFronzo RA. The insulin resistance syndrome: physiological considerations. Diab Vasc Dis Res. 2007;4:13-9. 
5. Kendall DM, Cuddihy RM, Bergenstal RM. Clinical application of incretin-based therapy: therapeutic potential, patient selection and clinical use. Eur J Intern Med. 2009;20:S329-39.

6. Meier JJ, Bonadonna RC. Role of reduced beta-cell mass versus impaired beta-cell function in the pathogenesis of type 2 diabetes. Diabetes Care. 2013;36(Suppl 2):S113-9.

7. American Diabetes Association. Standards of medical care in diabetes-2014. Diabetes Care. 2014;37(Suppl 1):S14-80.

8. American Association of Clinical Endocrinologists. AACE Comprehensive Diabetes Management Algorithm. http://www.aace.com/files/aace_ algorithm.pdf. Accessed Nov, 2013.

9. Byetta [package insert]. San Diego: Amylin Pharmaceuticals, LLC; 2011.

10. Victoza [package insert]. Bagsvaerd: Novo Nordisk A/S; 2011.

11. Bydureon [package insert]. San Diego: Amylin Pharmaceuticals, LLC; 2012.

12. Holst JJ. The physiology of glucagon-like peptide 1 . Physiol Rev. 2007;87:1409-39.

13. Drucker DJ. The biology of incretin hormones. Cell Metab. 2006;3:153-65.

14. Orskov C, Holst JJ, Nielsen OV. Effect of truncated glucagon-like peptide-1 [proglucagon-(78-107) amide] on endocrine secretion from pig pancreas, antrum, and nonantral stomach. Endocrinology. 1988;123:2009-13.

15. Nauck MA, Heimesaat MM, Behle K, Holst JJ, Nauck MS, Ritzel R, et al. Effects of glucagon-like peptide 1 on counterregulatory hormone responses, cognitive functions, and insulin secretion during hyperinsulinemic, stepped hypoglycemic clamp experiments in healthy volunteers. J Clin Endocrinol Metab. 2002;87:1239-46.

16. Nauck MA, Niedereichholz U, Ettler R, Holst JJ, Orskov C, Ritzel R, et al. Glucagon-like peptide 1 inhibition of gastric emptying outweighs its insulinotropic effects in healthy humans. Am J Physiol. 1997;273:E981-8.

17. Tang-Christensen M, Larsen PJ, Göke R, Fink-Jensen A, Jessop DS, Moller M, et al. Central administration of GLP-1-(7-36) amide inhibits food and water intake in rats. Am J Physiol. 1996;271:R848-56.

18. Lovshin JA, Drucker DJ. Incretin-based therapies for type 2 diabetes mellitus. Nat Rev Endocrinol. 2009;5:262-9.
19. Cersosimo E, Gastaldelli A, Cervera A, Wajcberg E, Sriwijilkamol A, Fernandez $M$, et al. Effect of exenatide on splanchnic and peripheral glucose metabolism in type 2 diabetic subjects. J Clin Endocrinol Metab. 2011;96:1763-70.

20. Cervera A, Wajcberg E, Sriwijitkamol A, Fernandez M, Zuo P, Triplitt C, et al. Mechanism of action of exenatide to reduce postprandial hyperglycemia in type 2 diabetes. Am J Physiol Endocrinol Metab. 2008;294:E846-52.

21. Kolterman OG, Buse JB, Fineman MS, Gaines E, Heintz S, Bicsak TA, et al. Synthetic exendin-4 (exenatide) significantly reduces postprandial and fasting plasma glucose in subjects with type 2 diabetes. J Clin Endocrinol Metab. 2003;88:3082-9.

22. Deacon CF, Johnsen AH, Holst JJ. Degradation of glucagon-like peptide-1 by human plasma in vitro yields an N-terminally truncated peptide that is a major endogenous metabolite in vivo. J Clin Endocrinol Metab. 1995;80:952-7.

23. DeYoung MB, MacConell L, Sarin V, Trautmann M, Herbert P. Encapsulation of exenatide in poly-(D, Llactide-co-glycolide) microspheres produced an investigational long-acting once-weekly formulation for type 2 diabetes. Diabetes Technol Ther. 2011;13:1145-54.

24. Shive MS, Anderson JM. Biodegradation and biocompatibility of PLA and PLGA microspheres. Adv Drug Deliv Rev. 1997;28:5-24.

25. Lewis DH. Controlled release of bioactive agents from lactide/glycolide polymers. In: Chasin $\mathrm{M}$, Langer RS, editors. Biodegradable polymers as drug delivery systems. New York: M. Dekker; 1990. p. 1-41.

26. Pillai CK, Sharma CP. Review paper: absorbable polymeric surgical sutures: chemistry, production, properties, biodegradability, and performance. J Biomater Appl. 2010;25:291-366.

27. Fineman M, Flanagan S, Taylor K, Aisporna M, Shen LZ, Mace KF, et al. Pharmacokinetics and pharmacodynamics of exenatide extended-release after single and multiple dosing. Clin Pharmacokinet. 2011;50:65-74.

28. Bergenstal RM, Wysham C, Macconell L, Malloy J, Walsh B, Yan P, et al. Efficacy and safety of exenatide once weekly versus sitagliptin or pioglitazone as an adjunct to metformin for treatment of type 2 diabetes (DURATION-2): a randomised trial. Lancet. 2010;376:431-9.

29. Blevins T, Pullman J, Malloy J, Yan P, Taylor K, Schulteis $\mathrm{C}$, et al. DURATION-5: exenatide once 
weekly resulted in greater improvements in glycemic control compared with exenatide twice daily in patients with type 2 diabetes. J Clin Endocrinol Metab. 2011;96:1301-10.

30. Diamant M, Van Gaal L, Stranks S, Northrup J, Cao D, Taylor $\mathrm{K}$, et al. Once weekly exenatide compared with insulin glargine titrated to target in patients with type 2 diabetes (DURATION-3): an open-label randomised trial. Lancet. 2010;375:2234-43.

31. Drucker DJ, Buse JB, Taylor K, Kendall DM, Trautmann M, Zhuang D, et al. Exenatide once weekly versus twice daily for the treatment of type 2 diabetes: a randomised, open-label, non-inferiority study. Lancet. 2008;372:1240-50.

32. Russell-Jones D, Cuddihy RM, Hanefeld M, Kumar A, González JG, Chan M, et al. Efficacy and safety of exenatide once weekly versus metformin, pioglitazone, and sitagliptin used as monotherapy in drug-naive patients with type 2 diabetes (DURATION-4): a 26-week double-blind study. Diabetes Care. 2012;35:252-8.

33. Buse JB, Nauck M, Forst T, Sheu WH, Shenouda SK, Heilmann CR, et al. Exenatide once weekly versus liraglutide once daily in patients with type 2 diabetes (DURATION-6): a randomised, open-label study. Lancet. 2013;381:117-24.

34. Trautmann M, Van Gaal L, Guerci B, Stranks S, Han J, Malloy J, et al. Exenatide once weekly: sustained glycemic and weight control through 3 years compared with insulin glargine. Diabetes. 2013;62:A17.

35. Sathyanarayana P, Jogi M, Muthupillai R, Krishnamurthy R, Samson SL, Bajaj M. Effects of combined exenatide and pioglitazone therapy on hepatic fat content in type 2 diabetes. Obesity (Silver Spring). 2011;19:2310-5.

36. Larsen PJ, Wulff EM, Gotfredsen CF, Brand CL, Sturis J, Vrang N, et al. Combination of the insulin sensitizer, pioglitazone, and the longacting GLP-1 human analog, liraglutide, exerts potent synergistic glucose-lowering efficacy in severely diabetic ZDF rats. Diabetes Obes Metab. 2008;10:301-11.

37. Abdul-Ghani MA, Puckett C, Adams J, Cersosimo E, Triplitt C, DeFronzo RA. Initial triple combination therapy is superior to stepwise add-on conventional therapy in newly diagnosed T2DM. 73rd Scientific Sessions of the American Diabetes Association, Chicago; 2013.

38. Tobin GS, Cavaghan MK, Hoogwerf BJ, McGill JB. Addition of exenatide twice daily to basal insulin for the treatment of type 2 diabetes: clinical studies and practical approaches to therapy. Int J Clin Pract. 2012;66:1147-57.

39. Buse JB, Bergenstal RM, Glass LC, Heilmann CR, Lewis MS, Kwan AY, et al. Use of twice-daily exenatide in basal insulin-treated patients with type 2 diabetes: a randomized, controlled trial. Ann Intern Med. 2011;154:103-12.

40. Cryer PE. Hypoglycemia, functional brain failure, and brain death. J Clin Invest. 2007;117:868-70.

41. Cryer PE, American Diabetes Association. Hypoglycemia in diabetes: pathophysiology, prevalence, and prevention. Alexandria: American Diabetes Association; 2009.

42. Cryer PE. Hypoglycemia. In: Melmed S, Williams $\mathrm{RH}$, editors. Williams textbook of endocrinology. 12th ed. Philadelphia: Elsevier/Saunders; 2011. p. $1552-81$.

43. Herman GA, Bergman A, Stevens C, Kotey P, Yi B, Zhao $P$, et al. Effect of single oral doses of sitagliptin, a dipeptidyl peptidase- 4 inhibitor, on incretin and plasma glucose levels after an oral glucose tolerance test in patients with type 2 diabetes. J Clin Endocrinol Metab. 2006;91: 4612-9.

44. Fineman MS, Shen LZ, Taylor K, Kim DD, Baron AD. Effectiveness of progressive dose-escalation of exenatide (exendin-4) in reducing dose-limiting side effects in subjects with type 2 diabetes. Diabetes Metab Res Rev. 2004;20:411-7.

45. Degn KB, Brock B, Juhl CB, Djurhuus CB, Grubert J, Kim D, et al. Effect of intravenous infusion of exenatide (synthetic exendin-4) on glucosedependent insulin secretion and counterregulation during hypoglycemia. Diabetes. 2004;53:2397-403.

46. Nissen SE, Wolski K. Effect of rosiglitazone on the risk of myocardial infarction and death from cardiovascular causes. N Engl J Med. 2007;356:2457-71.

47. Gerstein HC, Miller ME, Byington RP, Goff DC Jr, Bigger JT, Buse JB, et al. Effects of intensive glucose lowering in type 2 diabetes. N Engl J Med. 2008; 358:2545-59.

48. Bailey CJ. Interpreting adverse signals in diabetes drug development programs. Diabetes Care. 2013; 36:2098-106.

49. Darpo B, Philip S, MacConell L, Cirincione B, Mitchell M, Han J, et al. Exenatide at therapeutic and supratherapeutic concentrations does not prolong the QTc interval in healthy subjects. Br J Clin Pharmacol. 2013;75:979-89. 
50. Sager P, Darpö B, Han J, Kothare P, Linnebjerg H, Mitchell M, et al. Exenatide once weekly did not affect corrected QT interval in patients with type 2 diabetes. Diabetes; 2011. http://professional.diabetes.org/ Abstracts_Display.aspx?TYP=1\&CID=87603. Accessed Sep, 2013.

51. MacConell L, Brown C, Gurney K, Han J. Safety and tolerability of exenatide twice daily in patients with type 2 diabetes: integrated analysis of 5594 patients from 19 placebo-controlled and comparatorcontrolled clinical trials. Diabetes Metab Syndr Obes. 2012;5:29-41.

52. Butler PC, Elashoff M, Elashoff R, Gale EA. A critical analysis of the clinical use of incretin-based therapies: are the GLP-1 therapies safe? Diabetes Care. 2013;36:2118-25.

53. Nauck MA. A critical analysis of the clinical use of incretin-based therapies: the benefits by far outweigh the potential risks. Diabetes Care. 2013;36:2126-32.

54. Elashoff M, Matveyenko AV, Gier B, Elashoff R, Butler PC. Pancreatitis, pancreatic, and thyroid cancer with glucagon-like peptide-1-based therapies. Gastroenterology. 2011;141:150-6.

55. Nauck MA, Friedrich N. Do GLP-1-based therapies increase cancer risk? Diabetes Care. 2013;36(Suppl 2):S245-52.

56. Noel RA, Braun DK, Patterson RE, Bloomgren GL. Increased risk of acute pancreatitis and biliary disease observed in patients with type 2 diabetes: a retrospective cohort study. Diabetes Care. 2009;32:834-8.

57. Blomgren KB, Sundstrom A, Steineck G, Wiholm BE. Obesity and treatment of diabetes with glyburide may both be risk factors for acute pancreatitis. Diabetes Care. 2002;25:298-302.

58. Scirica BM, Bhatt DL, Braunwald E, Steg PG, Davidson J, Hirshberg B, et al. Saxagliptin and cardiovascular outcomes in patients with type 2 diabetes mellitus. N Engl J Med. 2013;369:1317-26.

59. White WB, Cannon CP, Heller SR, Nissen SE, Bergenstal RM, Bakris GL, et al. Alogliptin after acute coronary syndrome in patients with type 2 diabetes. N Engl J Med. 2013;369:1327-35.

60. Dore DD, Seeger JD, Chan KA. Use of a claims-based active drug safety surveillance system to assess the risk of acute pancreatitis with exenatide or sitagliptin compared to metformin or glyburide. Curr Med Res Opin. 2009;25:1019-27.

61. European_Medicines_Agency. Assessment report for GLP-1 based therapies. http://www.ema.
europa.eu/docs/en_GB/document_library/Report/ 2013/08/WC500147026.pdf. Accessed Jan, 2014.

62. Brooks M. FDA sides with EMA on incretin diabetes drugs. http://www.medscape.com/viewarticle/ 808830. Accessed Jan, 2014.

63. Copley K, McCowen K, Hiles R, Nielsen LL, Young A, Parkes DG. Investigation of exenatide elimination and its in vivo and in vitro degradation. Curr Drug Metab. 2006;7:367-74.

64. Tuttle KR, Heilmann C, Hoogwerf BJ, Brown C, Anderson PW. Effects of exenatide on kidney function, adverse events, and clinical end points of kidney disease in type 2 diabetes. Am J Kidney Dis. 2013;62:396-8.

65. Pencek R, Blickensderfer A, Li Y, Brunell SC, Chen S. Exenatide once weekly for the treatment of type 2 diabetes: effectiveness and tolerability in patient subpopulations. Int J Clin Pract. 2012;66: 1021-32.

66. Cramer JA. A systematic review of adherence with medications for diabetes. Diabetes Care. 2004;27:1218-24.

67. Dezii CM, Kawabata H, Tran M. Effects of oncedaily and twice-daily dosing on adherence with prescribed glipizide oral therapy for type 2 diabetes. South Med J. 2002;95:68-71.

68. Polonsky WH, Fisher L, Hessler D, Bruhn D, Best JH. Patient perspectives on once-weekly medications for diabetes. Diabetes Obes Metab. 2011;13:144-9.

69. Fonseca T, Clegg J, Caputo G, Norrbacka K, Dilla $\mathrm{T}$, Alvarez M. The cost-effectiveness of exenatide once weekly compared with exenatide twice daily and insulin glargine for the treatment of patients with type two diabetes and body mass index $>$ / $=30 \mathrm{~kg} / \mathrm{m}(2)$ in Spain. J Med Econ. 2013;16:926-38.

70. Beaudet A, Palmer JL, Timlin L, Wilson B, Bruhn D, Boye KS, et al. Cost-utility of exenatide once weekly compared with insulin glargine in patients with type 2 diabetes in the UK. J Med Econ. 2011;14:357-66.

71. Brandle M, Erny-Albrecht KM, Goodall G, Spinas GA, Streit P, Valentine WJ. Exenatide versus insulin glargine: a cost-effectiveness evaluation in patients with Type 2 diabetes in Switzerland. Int J Clin Pharmacol Ther. 2009;47:501-15.

72. Samyshkin Y, Guillermin AL, Best JH, Brunell SC, Lloyd A. Long-term cost-utility analysis of exenatide once weekly versus insulin glargine for the treatment of type 2 diabetes patients in the US. J Med Econ. 2012;15(Suppl 2):6-13. 
73. Guillermin AL, Lloyd A, Best JH, DeYoung MB, Samyshkin Y, Gaebler JA. Long-term costconsequence analysis of exenatide once weekly vs sitagliptin or pioglitazone for the treatment of type
2 diabetes patients in the United States. J Med Econ. 2012;15:654-63. 